\title{
Cell-Laden Thermosensitive Chitosan Hydrogel Bioinks for 3D Bioprinting Applications
}

\author{
Jongbeom Ku ${ }^{1,+}{ }^{\dagger}$, Hoon Seonwoo ${ }^{2,+}$, Sangbae Park ${ }^{1}{ }^{\oplus}$, Kyoung-Je Jang ${ }^{1}$, Juo Lee ${ }^{2}$, \\ Myungchul Lee ${ }^{1}$, Jae Woon Lim ${ }^{1}$, Jangho Kim ${ }^{3}$ and Jong Hoon Chung ${ }^{1,4, *(D)}$ \\ 1 Department of Biosystems \& Biomaterials Science and Engineering, Seoul National University, \\ Seoul 151-742, Korea; kjb0407@snu.ac.kr (J.K.); sb92park@snu.ac.kr (S.P.); trudwp04@snu.ac.kr (K.-J.J.); \\ lmcjuice@snu.ac.kr (M.L.); jwlim1130@snu.ac.kr (J.W.L.) \\ 2 Department of Industrial Machinery Engineering, College of Life Sciences and Natural Resources, Sunchon \\ National University, 255, Jungang-ro, Suncheon-si, Jeollanam-do 57922, Korea; dlwndh10@hanmail.net (J.L.); \\ uhun906@gmail.com (H.S.) \\ 3 Department of Rural and Biosystems Engineering, Chonnam National University, Gwangju 500-757, Korea; \\ rain2000@jnu.ac.kr \\ 4 Research Institute of Agriculture and Life Sciences, Seoul National University, Seoul 151-742, Korea \\ * Correspondence: jchung@snu.ac.kr; Tel.: +82-2-880-4601 \\ + These two authors equally contributed to this study.
}

Received: 9 March 2020; Accepted: 1 April 2020; Published: 3 April 2020

\begin{abstract}
Three-dimensional (3D) bioprinting is a technology used to deposit cell-laden biomaterials for the construction of complex tissues. Thermosensitive hydrogels are physically cross-linked by non-covalent interaction without using crosslinkers, facilitating low cytotoxicity and cell viability. Chitosan, which is a non-toxic, biocompatible and biodegradable polysaccharide, can be used as a thermosensitive hydrogel. Therefore, chitosan hydrogel could be of potential use as a 3D bioprinting ink. The purpose of this study was to develop and compare the effectivity of different bioinks based on chitosan hydrogels for 3D bioprinting. The solvent type did not affect the gel shape and gelation time, whereas acetic acid exhibited better biocompatibility compared to lactic and hydrochloric acids. The nature of the gelling agent was found to have a stronger influence on these characteristics than that of the solvent. The $\mathrm{NaHCO}_{3}$ moiety exhibited a higher growth rate of the storage modulus $\left(\mathrm{G}^{\prime}\right)$ and a more irregular porous structure than that of the $\beta$-glycerophosphate $(\beta-\mathrm{GP})$ and $\mathrm{K}_{2} \mathrm{HPO}_{4}$ groups. Cell viability, and live and dead assays, showed that the $\mathrm{NaHCO}_{3}$ group was more efficient for cell adhesion. The type of gelling agent did not lead to appreciable differences in cell-laden constructs. The $\mathrm{NaHCO}_{3}$ group was more amenable to bioprinting, compared to the $\beta-\mathrm{GP}$ and $\mathrm{K}_{2} \mathrm{HPO}_{4}$ groups. The chitosan hydrogel bioinks could, therefore, be good candidates for 3D bioprinting and would pave the way for patient-specific regenerative medicines.
\end{abstract}

Keywords: 3D bioprinting; bioink; hydrogel; chitosan

\section{Introduction}

Three-dimensional (3D) printing, also known as additive manufacturing, can be used to create a solid 3D structure through a layer-by-layer process. At present, the 3D printing of biocompatible materials represents an active area of research and constitutes tools for tissue engineering and regenerative medicine [1]. In the early years of the application of 3D printing in the fields of tissue engineering and regenerative medicine, 3D printing was applied to fabricate 3D scaffolds without cells. However, the development of 3D printing technology has led to the formation of complex tissue constructs through the deposition of cells and hydrogels via a layer-by-layer process called $3 \mathrm{D}$ bioprinting [2]. Cells and hydrogels have been generated from printable, non-toxic bioinks [2-4]. 
The major technologies used for 3D bioprinting include inkjet bioprinting, laser-assisted bioprinting, digital light processing (DLP) bioprinting, and extrusion bioprinting [3]. Inkjet bioprinting is a modification of conventional two-dimensional (2D) inkjet printing, and was the first bioprinting technology to be developed. Inkjet bioprinters use thermal or piezoelectric actuators to eject liquid droplets onto substrates [5]. The advantages of inkjet bioprinters include their relatively low cost and high-speed printing, although there is a risk that cells and materials generated this way are exposed to thermal and mechanical stress and nozzle clogging. Laser-assisted bioprinting is less common than inkjet or microextrusion bioprinting. However, laser-assisted bioprinters are being used in cell printing, tissue engineering and regenerative medicine. Laser-assisted bioprinters consist of a 'ribbon' structure that contains an energy absorbing layer on the top and a layer of liquid bioink solution suspended at the bottom [6]. During printing, a focused laser pulses on the absorbing layer of the ribbon to create a high-pressure bubble that stimulates bioink flow toward the collector substrate. Laser-assisted bioprinters do not exert any mechanical stress on cells, which results in high cell viability. Moreover, nozzle-free devices could be used to circumvent the nozzle clogging problem. Despite these advantages, laser-assisted bioprinters are not used commonly owing to their high cost and complex device setup. DLP bioprinters are based on a dynamic optical projection stereolithography (DOPsL) platform that projects an optical pattern using UV light [7]. Compared to other 3D printers, the DLP bioprinter constructs a structure by continuously projecting the plane of the optical pattern onto the photopolymer vat. The DLP bioprinter has a high printing speed and cell viability, but it is not widely used owing to its high cost of operations [2,3]. Extrusion bioprinters commonly contain pneumatic or mechanical (piston or screw) dispensing systems, which deposit biomaterials onto substrates [8]. Almost all commercially available bioprinters are based on the extrusion model. Extrusion bioprinters are suitable for a wide range of hydrogels with different viscosities that exhibit compatible photochemical and thermal crosslinking properties. The cell viability associated with extrusion bioprinters is lower than that with inkjet ones, but the former are capable of depositing very high cell densities at a reasonable cost [2]. Based on currently available technology, only extrusion bioprinters are capable of 3D printing of a wide range of materials [9], rendering their use for 3D bioprinting important.

In the field of 3D bioprinting, bioinks such as hydrogels or biomaterials could be printed onto substrates to facilitate the attachment of cells. There are several types of bioinks available, including collagen, gelatin, Matrigel ${ }^{\mathrm{TM}}$, agarose, and alginate [10]. These materials are usually amenable to printing owing to their biocompatibility, nontoxic degradation kinetics and byproducts, and structural and mechanical properties suitable for printing and material biomimicry [2]. However, most hydrogels use crosslinkers that could induce cell death [11]. In contrast, thermosensitive hydrogels, which are liquid at room temperature and transit into gels as the temperature increases (Sol-gel transition) [12], could be crosslinked physically (without chemical crosslinkers). Thus, thermosensitive hydrogels could overcome the limitations associated with cell viability and cytotoxicity. Biomaterials derived from natural resources are preferred over those made of synthetic polymers for bioprinting. Among these, chitosan, which has been widely used in tissue engineering owing to its biocompatibility and osteoconductivity, could be used as a thermosensitive hydrogel. In fact, chitosan hydrogels are presently used for the 3D printing of biomaterials $[13,14]$. The gelling agent and solvent in chitosan-based thermosensitive hydrogels play an important role in the sol-gel transition [15]. Dang et al. suggested that hydrochloric acid, lactic acid, and acetic acid are suitable solvents for chitosan-based thermosensitive hydrogels [16]. Furthermore, the use of $\beta$-glycerophosphate ( $\beta$-GP), potassium phosphate $\left(\mathrm{K}_{2} \mathrm{HPO}_{4}\right)$, and sodium bicarbonate $\left(\mathrm{NaHCO}_{3}\right)$ as gelling agents has been reported previously $[17,18]$. However, there is no study on the comparative suitability of these gelling agents or solvents for 3D bioprinting.

The objectives of this study were to develop bioinks from chitosan hydrogels for the purpose of 3D bioprinting (Figure 1A) and to compare the potential suitability of the developed chitosan hydrogel as a bioink. In addition, the optimal solvents and gelling agents for the fabrication, characterization, printability, and biocompatibility of chitosan bioinks (CBIs) were explored. 
A

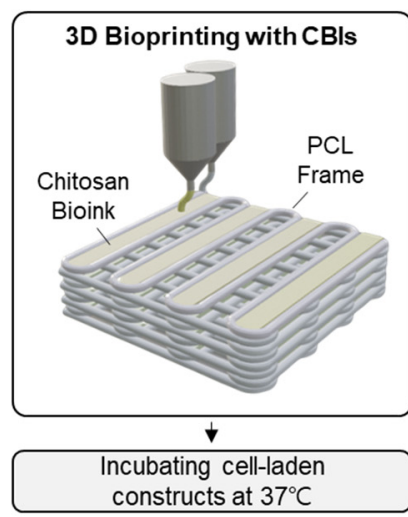

C

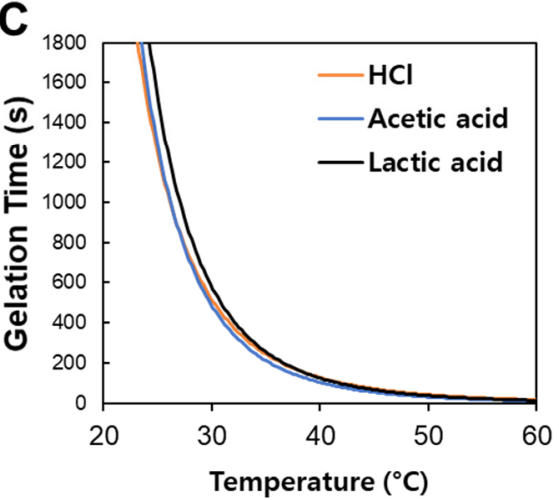

B
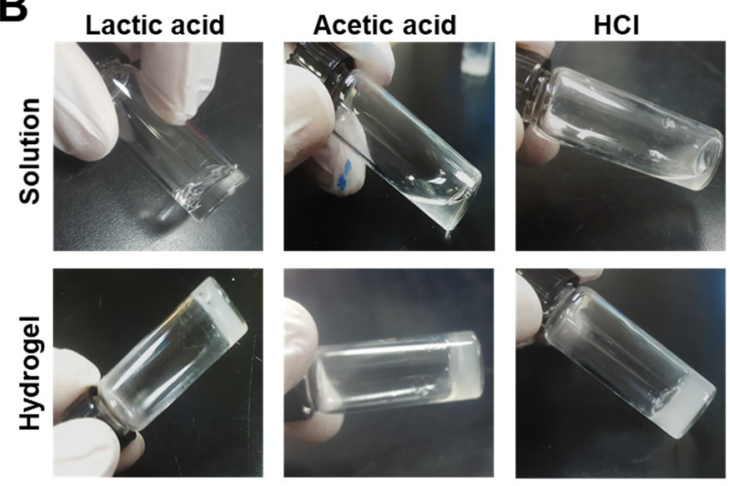

D

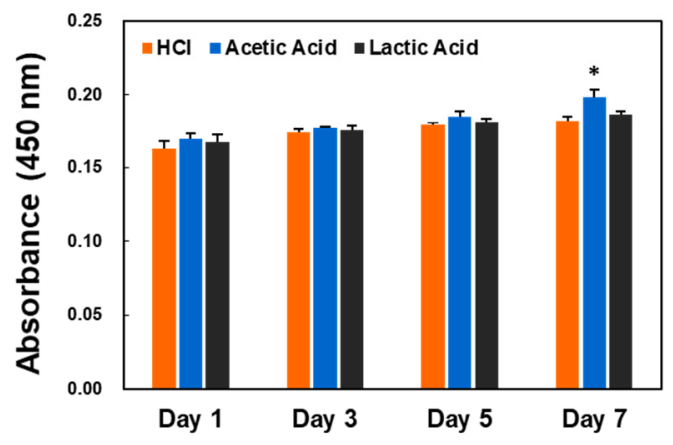

Figure 1. Characteristics of the chitosan hydrogels. (A) Schematic representation of the 3D bioprinter for 3D printing and gelation using CBIs. (B) Representative images of the chitosan solution and hydrogel in the presence of different solvents. (C) Gelation times of the chitosan hydrogel solution in different solvents. The test tube inversion method was used to determine the gelation time. (D) Cell viability of PDLSCs via indirect culture estimated using the WST assay. Error bars indicate standard deviation ( $\mathrm{n}=5$ for each group). ${ }^{*}$ Represents a statistically significant difference compared to the control group $(p<0.05)$. CBIs, chitosan bioinks; PDLSCs, periodontal ligament stem cells; WST, water soluble tetrazolium salt.

\section{Materials and Methods}

\subsection{Preparation of the Chitosan Hydrogel}

The chitosan hydrogel solution was prepared as described before [17-19]. A 1.67\% (w/v) chitosan (CS, 95\% or greater degree of deacetylation, YB Bio, Yeongdeok, Korea) solution was prepared by stirring powdered chitosan in $0.1 \mathrm{M}$ aqueous acetic acid at room temperature overnight. The insoluble particles in the chitosan solution were then filtered. The $56 \%(w / v) \beta$-glycerophosphate ( $\beta$-GP, Sigma-Aldrich, St. Louis, MO, USA), $8 \%$ Potassium phosphate $\left(\mathrm{K}_{2} \mathrm{HPO}_{4}\right.$, Duksan, Ansan, Korea) and $3.5 \%$ Sodium bicarbonate $\left(\mathrm{NaHCO}_{3}\right.$, Duksan, Ansan, Korea) solution was prepared in an $\alpha$-minimum essential medium ( $\alpha$-MEM, Welgene Inc., Gyeongsan, Korea) for use as a gelling agent, sterilized using syringe filters with $0.2 \mathrm{~mm}$ pore size and stored at $4{ }^{\circ} \mathrm{C}$. The chitosan hydrogel solution was prepared by adding the gelling agent solution to the chitosan solution at a 9:1 ratio, followed by $\mathrm{pH}$ neutralization using $1 \mathrm{M}$ of the $\mathrm{NaOH}$ solution (Duksan, Ansan, Korea). In order to avoid precipitation of the chitosan, a $\mathrm{NaOH}$ solution was gradually added until the $\mathrm{pH}$ of the chitosan solution reached above 6.5 . 


\subsection{Characterization of the Chitosan Hydrogel}

\subsubsection{Gelation Time Determination}

The gelation time of each chitosan hydrogel was measured at constant temperatures of 24, 37, 50 and $70{ }^{\circ} \mathrm{C}$ in a water bath using the test tube inversion method [20]. The time measurement was initiated after the sample was incubated in a tank at the predetermined temperature. The fluidity of the sample was tracked by tilting the tube every $30 \mathrm{~s}$. The gelation time was estimated as the time point when the sample flow stopped completely.

\subsubsection{Rheological Analysis}

A rheological analysis was performed with an advanced rheometric expansion system (ARES, Rheometric Scientific, UK). Changes in the elastic (storage) modulus $\left(\mathrm{G}^{\prime}\right)$ and the viscous (loss) modulus $\left(G^{\prime \prime}\right)$ were recorded as a function of the temperature $\left(25-50^{\circ} \mathrm{C}\right)$ at a fixed frequency of $1 \mathrm{~Hz}$. The temperature was varied with a constant heating rate of $1^{\circ} \mathrm{C} / \mathrm{min}$.

\subsubsection{Field Emission Scanning Electron Microscopy}

The shape and surface morphology of chitosan hydrogels were explored using a field emission scanning electron microscope (Carl Zeiss, Oberkochen, Germany). The chitosan hydrogels were prepared at $37^{\circ} \mathrm{C}$ for $3 \mathrm{~h}$ and then kept dipped into distilled water at $37^{\circ} \mathrm{C}$ for 1 day. Next, the samples were lyophilized overnight with a freeze dryer (IlshinBiobase, Dongducheon, Korea).

\subsection{Biocompatibility of Chitosan Bioinks (CBIs)}

\subsubsection{Cell Culture}

Human periodontal ligament stem cells (PDLSCs) were isolated from the adult patients' dental tissue for treatment purposes (Intellectual Biointerface Engineering Center, Dental Research Institute, College of Dentistry, Seoul National University) as described in our previous work. All experiments related to the PDLSCs were approved by the Seoul National University Institutional Animal Care and Use Committee (SNU-120427-2-2) [21]. The cells were cultured with growth-conditioned $\alpha$-MEM supplemented with 10\% fetal bovine serum (FBS, Welgene Inc., Gyeongsan, Korea) and 1\% antibiotics (Welgene Inc., Gyeongsan, Korea). The cells were cultured in a humidified incubator with $5 \% \mathrm{CO}_{2}$ at $37^{\circ} \mathrm{C}$.

\subsubsection{Water Soluble Tetrazolium Salt (WST) Assay}

The biological effects of chitosan hydrogels were examined by water soluble tetrazolium salt (WST, Daeil Lab Inc., Seoul, Korea) assay. The PDLSCs were cultured in $\alpha$-MEM containing $1 \%$ antibiotics and $10 \% \mathrm{FBS}$, as described in the previous section. Aliquots of $0.5 \mathrm{~mL}$ of the hydrogel solutions were pipetted into 24-well plates (Nunc ${ }^{\mathrm{TM}}$, Roskilde, Denmark) and allowed to undergo the sol-to-gel transition at $37^{\circ} \mathrm{C}$ for $1 \mathrm{~h}$ prior to the cell culture. $250 \mu \mathrm{L}$ of PDLSC-containing media were pipetted into each well. Cells were seeded at a density of 20,000 cells/well. After 1,3, 5 and 7 days, cell viability was determined using a microplate reader.

\subsubsection{Live and Dead Assay}

PDLSCs with chitosan hydrogels were seeded on 24-well plates at a density of 20,000 cells/well and incubated for 7 days. Viable cells were stained with the live and dead cell assay kit (Abcam, Cambridge, UK) and cell morphology was observed on days 1 and 7. 


\subsection{D Bioprinting Cell-Laden Constructs}

\subsubsection{D Bioprinter}

A 3D bioprinting system consisting of a motorized xyz-stage, a heating bed and a multi-nozzle printer head was developed (Figure 1A). The multi-nozzle printer head was composed of one filament extruder for polymers and four customized syringe pump dispensers for hydrogel printing. The open source Repetier-Firmware software was modified and utilized for system operations. The 3D bioprinting system was operated on a bio-safety clean bench (Hanbaek, Bucheon, Korea) in order to prevent contamination during the printing process.

\subsubsection{D Bioprinting of Polycaprolactone and CBI Constructs}

The 3D bioprinting system developed in our lab was used for printing the cell-laden constructs. Polycaprolactone (PCL, Mw 43000, Polysciences, Warrington, PA, USA) was printed as the framework using the filament extruder at a $65^{\circ} \mathrm{C}$ head temperature. Customized syringe pumps that can be adjusted for the set value of materials were used to print the cell-laden chitosan hydrogel. The PDLSCs were encapsulated at a concentration of $5 \times 10^{5}$ cells $/ \mathrm{mL}$ in the chitosan hydrogel solution, followed by the transfer of the cell-laden chitosan hydrogel to a sterile disposable syringe. The filament extruder and mechanical dispensing system were alternately used to fabricate a cell-laden construct. The lattice-type structure was of a thickness of $2 \mathrm{~mm}$ with an 8-layer height. At the end of the printing process, gelation of the cell-laden construct was induced by incubation at $37^{\circ} \mathrm{C}$.

\subsubsection{Cell Viability of Cell-Laden Constructs}

Cells experienced a relatively high shear-stress during the printing process. Therefore, we verified the viability of the cells after printing, using the live and dead cell assay, as described above.

\subsection{Statistical Data Analysis}

Statistical analysis was carried out using the statistical analysis system (SAS) for Windows v9.4 (SAS Institute, Inc., Cary, NC, USA). The statistical significance between the control and treatment groups was compared with a one-way ANOVA at $p<0.05$. The data were reported as a mean \pm standard deviation, $\mathrm{n}=5$.

\section{Results and Discussion}

\subsection{Effect of Solvents on Chitosan Hydrogels}

The characteristics of the CBIs based on the solvents used were investigated first. Figure 1B shows that the chitosan hydrogel solution was transformed to the gel phase upon incubation at $37^{\circ} \mathrm{C}$. The sol-gel transition was marked by the solution becoming turbid. All groups easily formed the hydrogel. Figure $1 \mathrm{C}$ shows that the gelation time decreased exponentially with the increasing temperature. The similar gelation time of chitosan hydrogels dissolved in different solvents indicated that they were almost similar in properties. Therefore, the solvent was not a key factor influencing the gelation time.

The effect of the chitosan hydrogel on the PDLSCs was estimated using the WST assay and cell morphology was observed under a microscope (Figure 1D). The WST assay showed that there was no significant difference in cell viability until day five. However, PDLSCs on the chitosan hydrogel dissolved in acetic acid had a significantly higher cell viability after day seven $(p<0.05)$. This result indicates that acetic acid has lower cytotoxicity compared to lactic and hydrochloric acids. Based on these experiments, acetic acid was chosen as the solvent for CBIs.

\subsection{Effects of Gelling Agents on Chitosan Hydrogels}

The evaluation of the effects of solvents on the chitosan hydrogels was followed by an exploration of the influence of different gelling agents. Figure 2A shows that regardless of the type of gelling agent, 
the chitosan solution transitioned into the gel state after incubation at $37^{\circ} \mathrm{C}$. The $\mathrm{CS} / \mathrm{K}_{2} \mathrm{HPO}_{4}$ group was associated with precipitates of large sizes that might induce clogging of the precision nozzles used in the 3D bioprinting process, since the precision nozzles used to extrude materials are very easily clogged [22]. On the other hand, the $\mathrm{CS} / \beta$-GP and $\mathrm{CS} / \mathrm{NaHCO}_{3}$ groups showed smaller-sized precipitates, compared to the $\mathrm{CS} / \mathrm{K}_{2} \mathrm{HPO}_{4}$ group. Figure $2 \mathrm{~B}$ shows the gelation time of chitosan hydrogels in the presence of different gelling agents. At higher temperatures, the shortest gelation time observed in this experiment was $20 \mathrm{~s}$. The $\mathrm{CS} / \mathrm{K}_{2} \mathrm{HPO}_{4}$ and $\mathrm{CS} / \mathrm{NaHCO}_{3}$ groups had the fastest and slowest gelation times, respectively. These results indicate that the $\mathrm{CS} / \mathrm{NaHCO}_{3}$ group could decrease the risk of nozzle clogging during the printing because it had the slowest gelation time. With an increase in temperature from $25^{\circ} \mathrm{C}$ to $50{ }^{\circ} \mathrm{C}$ (Figure 2D), all samples exhibited a rapid increase in the storage modulus $\left(\mathrm{G}^{\prime}\right)$ and a decrease in $\tan (\delta)$, showing that the sol-gel phase transition occurred around $37^{\circ} \mathrm{C}$. Figure $2 \mathrm{E}$ shows the microstructure of the chitosan hydrogels. The inner structure has interconnected pores of irregular sizes. Pores larger than 20-100 $\mu \mathrm{m}$ have been reported to help cell penetration and pores with a size larger than $100 \mu \mathrm{m}$ have been found to be associated with neovascularization, since these could provide a large enough space. Therefore, chitosan hydrogels may support cellular dispersions and neovascularization. The $\mathrm{CS} / \mathrm{K}_{2} \mathrm{HPO}_{4}$ group had a smaller pore size, whereas the $\mathrm{CS} / \beta-\mathrm{GP}$ and $\mathrm{CS} / \mathrm{NaHCO}_{3}$ groups had larger pores. Therefore, the $\mathrm{CS} / \beta-\mathrm{GP}$ and $\mathrm{CS} / \mathrm{NaHCO}_{3}$ groups might support cell dispersion and neovascularization better than the $\mathrm{CS} / \mathrm{K}_{2} \mathrm{HPO}_{4}$ group.

A

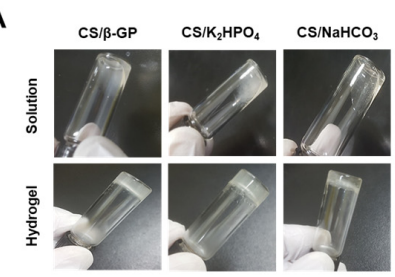

B

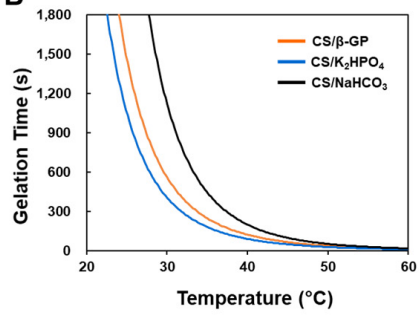

C

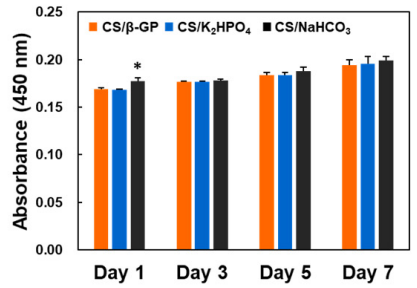

D

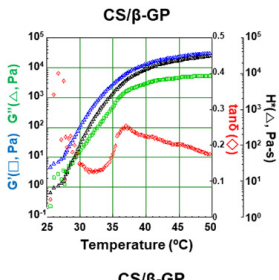

CS/ß-GP

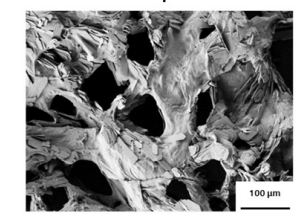

$\mathbf{F}$
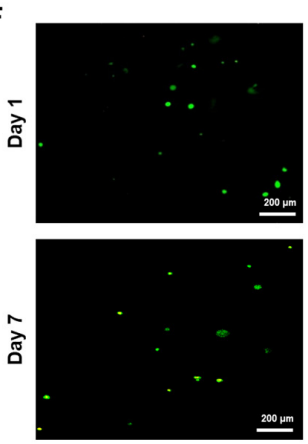

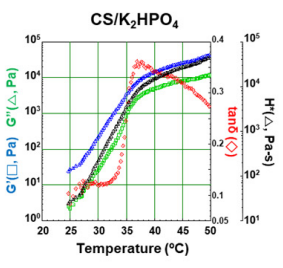

$\mathrm{CS} / \mathrm{K}_{2} \mathrm{HPO}_{4}$
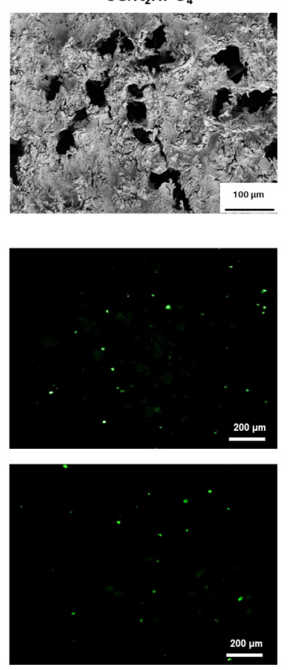

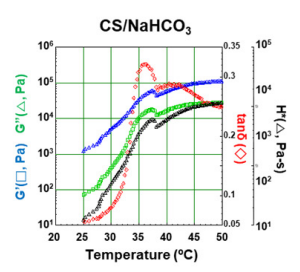

$\mathrm{CS} / \mathrm{NaHCO}_{3}$
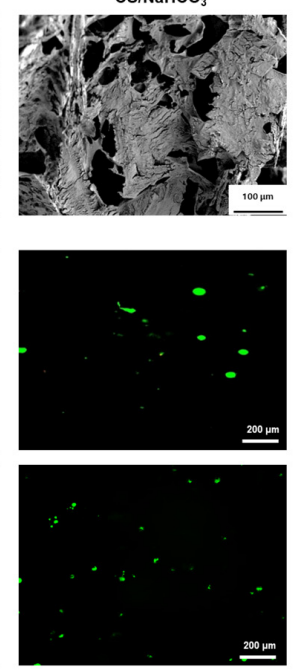

Figure 2. Influence of gelling agents on chitosan hydrogels. (A) Representative images of chitosan sol-gel transitions induced by temperature. (B) Gelation time of chitosan hydrogels in the presence of different gelling agents, as estimated by the test tube inversion method. (C) Cell viability of PDLSCs via indirect culture using the WST- 1 assay. Error bars indicate standard deviation ( $\mathrm{n}=5$ for each group). * Represents statistically significant differences compared to the control group $(p<0.05)$. (D) Rheological analysis of chitosan hydrogels, with temperature-dependent changes in the storage modulus $\left(\mathrm{G}^{\prime}\right)$ and loss modulus (G") from $25^{\circ} \mathrm{C}$ to $50{ }^{\circ} \mathrm{C}$. (E) SEM images of the surface of the chitosan hydrogel. Samples were prepared by dipping chitosan gels in distilled water at $37^{\circ} \mathrm{C}$ for 1 day, followed by lyophilization overnight. (F) Live and dead assay results of PDLSCs encapsulated in chitosan hydrogels (green: live cells; red: dead cells). PDLSCs, periodontal ligament stem cells; SEM, scanning electron microscopy; WST, water soluble tetrazolium salt. 
An in vitro assay using PDLSCs demonstrated the cytocompatibility of the chitosan hydrogels (Figure 2C). On day 1 , the $\mathrm{CS} / \mathrm{NaHCO}_{3}$ group showed higher cell adhesion rate compared to the other groups. However, on day seven, there was no significant difference among chitosan hydrogels containing different gelling agents. Chitosan hydrogels are, therefore, a potential candidate for bioink production due to their biocompatibility. Figure $2 \mathrm{~F}$ shows representative images from the live and dead assay. These results demonstrate that cells encapsulated in chitosan hydrogels were viable and well-dispersed, with a round morphology. After incubation for seven days, cells were more dispersed than after a one day incubation, with higher cell viability. This suggests that cell encapsulation with chitosan hydrogels is associated with minimal cytotoxicity, provided the cells were well-dispersed during the incubation. The concentration of the gelling agent appears to be the important factor influencing cell viability. Therefore, a $56 \%(w / v) \beta-G P, 8 \% \mathrm{~K}_{2} \mathrm{HPO}_{4}$, or $3.5 \% \mathrm{NaHCO}_{3}$ solution could be suitable for culturing cells.

\subsection{D Printing Cell-Laden Constructs}

The printability of CBIs was investigated using the 3D bioprinting system (Figure 3A). Figure 3B shows that the CBIs successfully printed a monolayer of a grid structure. The biocompatibility was established using the live and dead assay. However, the printed CBIs did not have enough rigidity to support the building of multilayers. Therefore, we fabricated grid structures that could encapsulate CBIs laden with cells (Figure 3C). An appropriate 3D printing method was used to load the cell-laden chitosan (pre-gel) solution into the PCL framework placed on petri dishes. One crucial aspect for the successful 3D bioprinting of cell-laden CBIs was the circumvention of nozzle clogging. The three kinds of gelling agents used in this study have similar printability (Figure 3D). As reported in the experiments for estimating gelation times, the $\mathrm{CS} / \mathrm{NaHCO}_{3}$ group had the shortest gelation time. Therefore, the use of the $\mathrm{CS} / \mathrm{NaHCO}_{3}$ group in $3 \mathrm{D}$ bioprinting might reduce the probability of nozzle clogging. In Figure 3E, the lattice-type structures generated by PCL are shown. CBIs could encapsulate PDLSCs and maintain high cell viability during the 3D bioprinting, both on days one and seven. PCL and CBI constructs are, therefore, suitable for long-term cell culture without any harmful side effects, regardless of the gelling agent used.

Chitosan has been widely used as a biomaterial in various forms, e.g., films [23,24], 3D scaffolds $[25,26]$, nanopatterned composites [27-29], and as wound healing reagents [30,31]. However, most chitosan-based materials have pre-formed shapes and specific treatments are required to transform their shapes to complement those of the wounds. Three-dimensional bioprinting is a revolutionary method for the generation of patient-specific scaffolds. The development of 3D printing bioinks is an important aspect of successful bioprinting. Because chitosan is characterized by antibiosis, biocompatibility, and biodegradability, it could be a superior candidate for 3D bioprinting inks. In this study, we introduced three types of CBIs based on different gelling agents. These have similar characteristics, except for their gelling times and precipitation regime. Among these 3 types, $\mathrm{CS} / \mathrm{NaHCO}_{3}$ was found to be the most appropriate 3D bioprinting material due to lesser nozzle clogging and good biocompatibility. Therefore, $\mathrm{CS} / \mathrm{NaHCO}_{3}$ bioink is suitable for $3 \mathrm{D}$ bioprinting and tissue engineering applications. By adding other components [32], such as growth factors [33] and graphene [21], their functionality could be enhanced, thereby extending their potential applicability. 
A

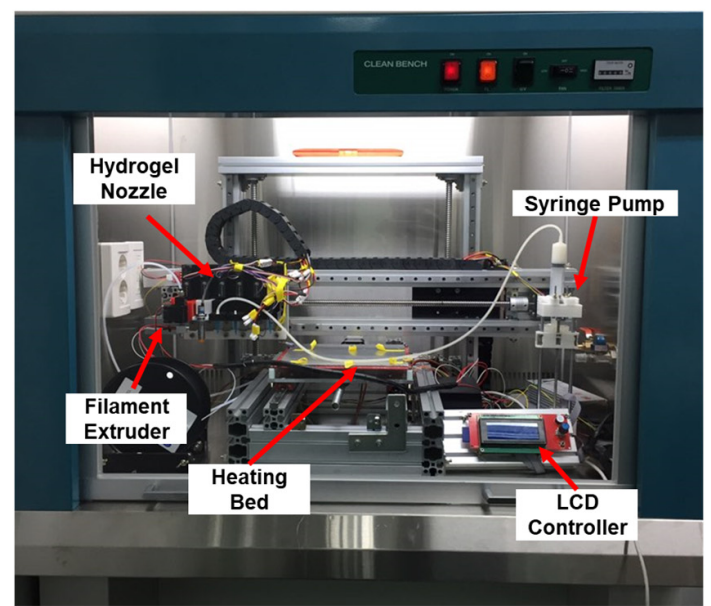

D $\quad C S / \beta-G P$
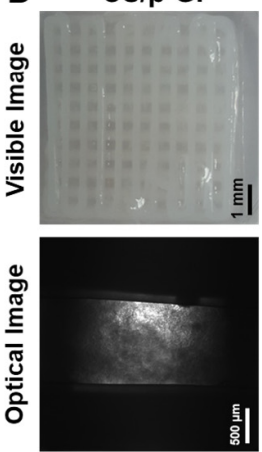

$\mathrm{CS} / \mathrm{K}_{2} \mathrm{HPO}_{4}$
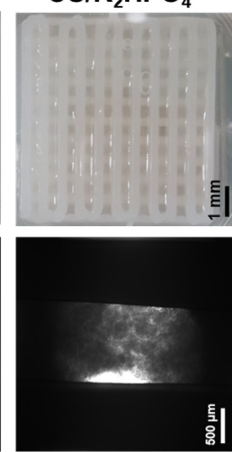
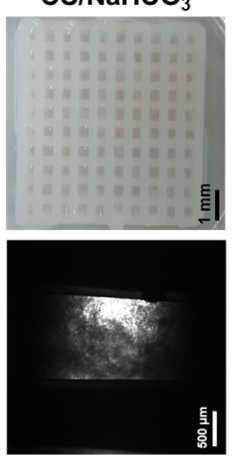

B
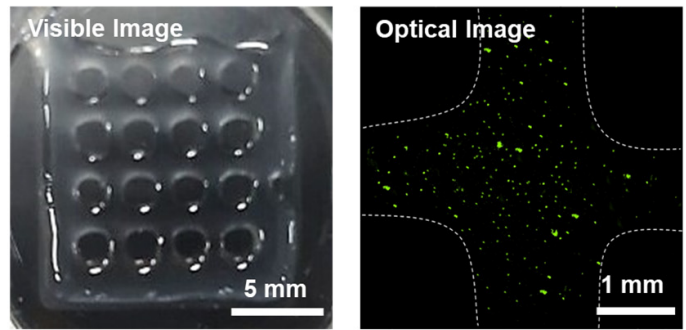

C
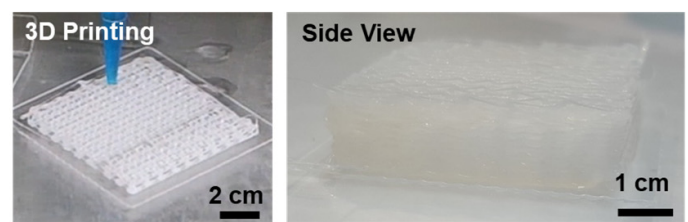

E

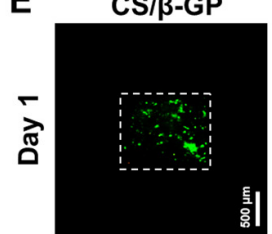

$\mathrm{CS} / \mathrm{K}_{2} \mathrm{HPO}_{4}$

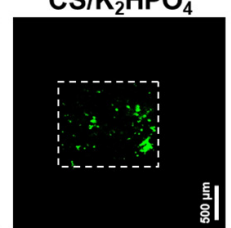

$\mathrm{CS} / \mathrm{NaHCO}_{3}$
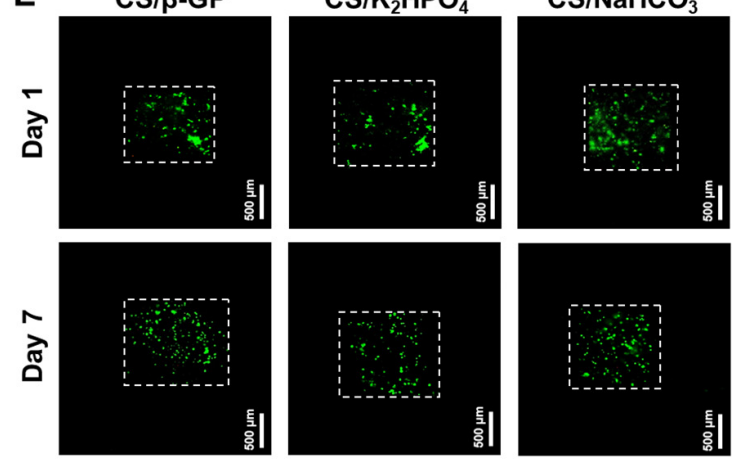

Figure 3. 3D printability of CBIs. (A) The 3D bioprinting system used in this study. (B) 3D bioprinting of cell-laden CBIs. (C) 3D bioprinting processes for the PCL and CBI constructs. (D) Representative microscopy images of 3D bioprinted structures using PCL and PDLSC-encapsulated CBIs. (E) Live and dead cell assay of 3D bioprinted cells (green: live cells; red: dead cells).

\section{Conclusions}

The objectives of this research were to develop and characterize CBIs amenable to 3D bioprinting using various solvents and gelling agents. Further, the cell viability and potential ability of the developed chitosan hydrogels as bioinks were investigated. These CBIs are anticipated to be useful in $3 \mathrm{D}$ printing applications in the field of tissue engineering and regenerative medicine.

Author Contributions: Conceptualization, J.K. (Jongbeom Ku), H.S., and J.H.C.; methodology, H.S., K.-J.J., and S.P.; experiment assistance, J.L., M.L., and J.W.L.; supervision and project administration, J.K. (Jangho Kim), H.S., and J.H.C. All authors have read and agreed to the published version of the manuscript.

Funding: This research was supported by the Basic Science Research Program through the National Research Foundation of Korea (NRF) and was funded by the Ministry of Education (NRF-2019R1I1A3A01064005 and NRF-2014R1A6A1030419). This research was also supported by Korea Institute of Planning and Evaluation for Technology in Food, Agriculture and Forestry (IPET) through Agri-Bio industry Technology Development Program, funded by Ministry of Agriculture, Food and Rural Affairs(MAFRA)(116135-3).

Conflicts of Interest: The authors declare no conflict of interest.

\section{References}

1. Chia, H.N.; Wu, B.M. Recent advances in 3D printing of biomaterials. J. Biol. Eng. 2015, 9, 4. [CrossRef]

2. Murphy, S.V.; Atala, A. 3D bioprinting of tissues and organs. Nat. Biotechnol. 2014, 32, 773-785. [CrossRef]

3. Zhu, W.; Ma, X.; Gou, M.; Mei, D.; Zhang, K.; Chen, S. 3D printing of functional biomaterials for tissue engineering. Curr. Opin. Biotechnol. 2016, 40, 103-112. [CrossRef] 
4. Kang, H.-W.; Lee, S.J.; Ko, I.K.; Kengla, C.; Yoo, J.J.; Atala, A. A 3D bioprinting system to produce human-scale tissue constructs with structural integrity. Nat. Biotechnol. 2016, 34, 312-319. [CrossRef]

5. Saunders, R.; Derby, B. Inkjet printing biomaterials for tissue engineering: Bioprinting. Int. Mater. Rev. 2014, 59, 430-448. [CrossRef]

6. Zhang, X.; Zhang, Y. Tissue Engineering Applications of Three-Dimensional Bioprinting. Cell Biophys. 2015, 72, 777-782. [CrossRef]

7. Hribar, K.C.; Soman, P.; Warner, J.; Chung, P.; Chen, S. Light-assisted direct-write of 3D functional biomaterials. Lab Chip 2014, 14, 268-275. [CrossRef]

8. Jose, R.R.; Rodriguez, M.J.; Dixon, T.A.; Omenetto, F.G.; Kaplan, D.L. Evolution of Bioinks and Additive Manufacturing Technologies for 3D Bioprinting. ACS Biomater. Sci. Eng. 2016, 2, 1662-1678. [CrossRef]

9. Visser, J.; Peters, B.; Burger, T.J.; Boomstra, J.; Dhert, W.; Melchels, F.P.; Malda, J. Biofabrication of multi-material anatomically shaped tissue constructs. Biofabrication 2013, 5, 035007. [CrossRef]

10. Wang, S.; Lee, J.M.; Yeong, W.Y. Smart hydrogels for 3D bioprinting. Int. J. Bioprint. 2015, 1, 3-14. [CrossRef]

11. Van Miller, J.P.; Hermansky, S.J.; Losco, P.; Ballantyne, B. Chronic toxicity and oncogenicity study with glutaraldehyde dosed in the drinking water of Fischer 344 rats. Toxicology 2002, 175, 177-189. [CrossRef]

12. Jeong, B.; Kim, S.W.; Bae, Y.H. Thermosensitive sol-gel reversible hydrogels. Adv. Drug Deliv. Rev. 2012, 64, 154-162. [CrossRef]

13. Roehm, K.D.; Madihally, S.V. Bioprinted chitosan-gelatin thermosensitive hydrogels using an inexpensive 3D printer. Biofabrication 2017, 10, 015002. [CrossRef]

14. Demirtaş, T.T.; Irmak, G.; Gümüşderelioğlu, M. A bioprintable form of chitosan hydrogel for bone tissue engineering. Biofabrication 2017, 9, 035003. [CrossRef]

15. Zhou, H.; Jiang, L.J.; Cao, P.P.; Li, J.B.; Chen, X. Glycerophosphate-based chitosan thermosensitive hydrogels and their biomedical applications. Carbohydr. Polym. 2015, 117, 524-536. [CrossRef]

16. Dang, Q.F.; Zou, S.H.; Chen, X.; Liu, C.; Li, J.J.; Zhou, X.; Liu, Y.; Cheng, X.J. Characterizations of chitosan-based highly porous hydrogel-The effects of the solvent. J. Appl. Polym. Sci. 2012, 125, E88-E98. [CrossRef]

17. Liu, L.; Tang, X.; Wang, Y.; Guo, S. Smart gelation of chitosan solution in the presence of $\mathrm{NaHCO}_{3}$ for injectable drug delivery system. Int. J. Pharm. 2011, 414, 6-15. [CrossRef]

18. Ta, H.T.; Han, H.; Larson, I.; Dass, C.R.; Dunstan, D.E. Chitosan-dibasic orthophosphate hydrogel: A potential drug delivery system. Int. J. Pharm. 2009, 371, 134-141. [CrossRef]

19. Chenite, A.; Chaput, C.; Wang, D.; Combes, C.; Buschmann, M.; Hoemann, C.D.; Leroux, J.; Atkinson, B.; Binette, F.; Selmani, A. Novel injectable neutral solutions of chitosan form biodegradable gels in situ. Biomaterials 2000, 21, 2155-2161. [CrossRef]

20. Chung, Y.-M.; Simmons, K.L.; Gutowska, A.; Jeong, B. Sol-Gel Transition Temperature of PLGA-g-PEG Aqueous Solutions. Biomacromolecules 2002, 3, 511-516. [CrossRef]

21. Seonwoo, H.; Jang, K.-J.; Lee, D.; Park, S.; Lee, M.; Park, S.; Lim, K.-T.; Kim, J.; Chung, J.H. Neurogenic Differentiation of Human Dental Pulp Stem Cells on Graphene-Polycaprolactone Hybrid Nanofibers. Nanomaterials 2018, 8, 554. [CrossRef] [PubMed]

22. Ghorbanian, S.; Qasaimeh, M.A.; Akbari, M.; Tamayol, A.; Juncker, D. Microfluidic direct writer with integrated declogging mechanism for fabricating cell-laden hydrogel constructs. Biomed. Microdevices 2014, 16, 387-395. [CrossRef] [PubMed]

23. Seonwoo, H.; Kim, S.W.; Shin, B.; Jang, K.-J.; Lee, M.; Choo, O.-S.; Choi, M.-J.; Kim, J.; Lim, K.-T.; Jang, J.H.; et al. Latent stem cell-stimulating therapy for regeneration of chronic tympanic membrane perforations using IGFBP2-releasing chitosan patch scaffolds. J. Biomater. Appl. 2019, 34, 198-207. [CrossRef] [PubMed]

24. Tang, R.; Du, Y.; Fan, L. Dialdehyde starch-crosslinked chitosan films and their antimicrobial effects. J. Polym. Sci. Part B Polym. Phys. 2003, 41, 993-997. [CrossRef]

25. Kim, J.; Kim, S.W.; Choi, S.J.; Lim, K.-T.; Bin Lee, J.; Seonwoo, H.; Choung, P.-H.; Park, K.; Cho, C.-S.; Choung, Y.-H.; et al. A Healing Method of Tympanic Membrane Perforations Using Three-Dimensional Porous Chitosan Scaffolds. Tissue Eng. Part A 2011, 17, 2763-2772. [CrossRef]

26. Ikeda, T.; Ikeda, K.; Yamamoto, K.; Ishizaki, H.; Yoshizawa, Y.; Yanagiguchi, K.; Yamada, S.; Hayashi, Y. Fabrication and Characteristics of Chitosan Sponge as a Tissue Engineering Scaffold. BioMed Res. Int. 2014, 2014, 1-8. [CrossRef] 
27. Kim, J.; Kim, Y.-R.; Kim, Y.; Lim, K.-T.; Seonwoo, H.; Park, S.; Cho, S.-P.; Hong, B.H.; Choung, Y.-H.; Chung, J.H.; et al. Graphene-incorporated chitosan substrata for adhesion and differentiation of human mesenchymal stem cells. J. Mater. Chem. B 2013, 1, 933. [CrossRef]

28. Pavinatto, F.J.; Caseli, L.; Oliveira, O.N. Chitosan in Nanostructured Thin Films. Biomacromolecules 2010, 11, 1897-1908. [CrossRef]

29. Schiffman, J.D.; Schauer, C. Cross-Linking Chitosan Nanofibers. Biomacromolecules 2007, 8, 594-601. [CrossRef]

30. Azad, A.K.; Sermsintham, N.; Chandrkrachang, S.; Stevens, W.F. Chitosan membrane as a wound-healing dressing: Characterization and clinical application. J. Biomed. Mater. Res. 2004, 69, 216-222. [CrossRef]

31. Denkbaş, E.B.; Oztürk, E.; Ozdemir, N.; Keçeci, K.; Agalar, C. Norfloxacin-loaded chitosan sponges as wound dressing material. J. Biomater. Appl. 2004, 18, 291-303. [CrossRef]

32. Rai, M.; Yadav, A.; Gade, A. Silver nanoparticles as a new generation of antimicrobials. Biotechnol. Adv. 2009, 27, 76-83. [CrossRef] [PubMed]

33. Seonwoo, H.; Shin, B.; Jang, K.; Lee, M.; Choo, O.; Park, S.; Kim, Y.C.; Choi, M.; Kim, J.; Garg, P.; et al. Epidermal Growth Factor-Releasing Radially Aligned Electrospun Nanofibrous Patches for the Regeneration of Chronic Tympanic Membrane Perforations. Adv. Healthc. Mater. 2018, 8, 1801160. [CrossRef] [PubMed]

(C) 2020 by the authors. Licensee MDPI, Basel, Switzerland. This article is an open access article distributed under the terms and conditions of the Creative Commons Attribution (CC BY) license (http://creativecommons.org/licenses/by/4.0/). 